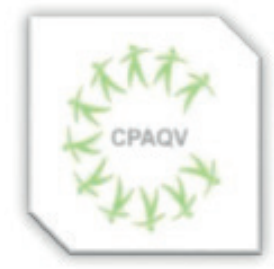

ISSN: 2178-7514

Vol. 12 | No. 1 | Ano 2020

ARTIGO ORIGINAL

\title{
IMAGEM CORPORAL, ESTADO NUTRICIONAL E NÍVEL DE ATIVIDADE FÍSICA DE ESTUDANTES DE EDUCAÇÃO FÍSICA
}

Body Image, Nutritional Status and Physical Activity Level of Physical Education Students

Silmara Aparecida de Sousa Ribeiro, Guanis de B. Vilela Junior ${ }^{2,3}$, Ricardo P. Passos ${ }^{2,3}$, Carlos H. P. Fileni ${ }^{2,3}$, Bráulio N. Lima ${ }^{2,3}$, José Ricardo L. Oliveira ${ }^{3}$, Gustavo C. Martins ${ }^{3}$, Luis Felipe Silio ${ }^{4}$, Tatiane Gomes Teixeira ${ }^{1}$, Silvia Teixeira de Pinho ${ }^{1}$, Angeliete Garcez Militao ${ }^{1}$, Daniel Delani ${ }^{1}$

\begin{abstract}
RESUMO
O objetivo do presente estudo foi comparar a percepção de Imagem Corporal (IC) Atual e da IC que se pretende ter com Índice de Massa Corporal (IMC), Percentual de Gordura e Prática de atividade física de estudantes de Educação Física. Com esse intuito o presente trabalho realizou uma pesquisa quanti-qualitativa, coletando dados de IMC, de percentual de gordura, nível de atividade física e de percepção de IC. Os dados foram reportados através de estatística descritiva, e mostram que estes estudantes de educação física não apresentam percepção de IC compatível com os dados apresentados pela avaliação do IMC e do percentual de gordura. A IC que se pretende ter, pode estar sofrendo influência de questões socioculturais que envolvem a estética, o mito do "corpo saudável" e a imagem de um "atleta" ou pessoa "muito treinada". Em geral, os dados encontrados neste estudo, sugerem que os estudantes de Educação Física praticam atividade física regularmente e essa prática está associada a uma expectativa em torno da IC, influenciando na IC que se pretende ter.
\end{abstract}

Palavras-chave: Imagem Corporal, Educação Física, Estado Nutricional e Nível de Atividade Física.

\section{ABSTRACT}

The aim of the present study was to compare the perception of Current Body Image (CI) and CI intended to have with Body Mass Index (BMI), Fat Percentage and Physical Activity Practice of Physical Education students. To this end, the present study conducted a quantitative and qualitative research, collecting data on BMI, fat percentage, level of physical activity and HF perception. The data were reported using descriptive statistics and show that these physical education students do not present CI perception compatible with the data presented by the BMI and fat percentage evaluation. The intended HF may be under the influence of sociocultural issues involving aesthetics, the myth of the "healthy body" and the image of an "athlete" or "very trained" person. In general, the data found in this study suggest that Physical Education students practice physical activity regularly and this practice is associated with an expectation around the CI, influencing the intended CI. and this practice is associated with an expectation around the BI, influencing the intended BI.

Keywords: Body Image, Physical Education, Nutritional Status and Physical Activity Level. 


\section{INTRODUÇÃO}

Atualmente as relações com o corpo são influenciadas por diversos fatores socioculturais. Estes fatores conduzem homens e mulheres a apresentarem um conjunto de preocupações e insatisfações com sua Imagem Corporal (IC), induzindo-os a se exercitarem, não com a finalidade de promoção à saúde, mas sim a preocupação com a estética, direcionando-os a desejos, hábitos e cuidado com a aparência visual do corpo ${ }^{(1)}$.

A IC pode ser definida como uma construção multidimensional que descreve amplamente as representações internas da estrutura corporal e da aparência física, em relação a nós mesmos e aos outros ${ }^{(1)}$.

A insatisfação com o corpo tem sido frequentemente associada à discrepância entre a percepção e o desejo relativo a um tamanho e a uma forma corporal $^{(2)}$. Embora constituam objetos complexos para investigações, existem evidências de que a mídia tem influências sobre os distúrbios na esfera da alimentação e da IC, pois ao mesmo tempo em que exigem "corpos perfeitos", paradoxalmente, estimula práticas alimentares não saudáveis.

O conflito da IC juntamente com a satisfação/ insatisfação pode resultar em distúrbio nutricional como, por exemplo, a obesidade e em distúrbios psicológicos como, a anorexia e bulimia ${ }^{(3)}$.

A obesidade e a desnutrição, quando associados à satisfação ou IC, estão correlacionados, pois os dois casos são reflexos da inconformidade com a própria aparência ${ }^{(4)}$.

Estudantes de Educação Física destacam-se por apresentarem peculiaridades com relação a IC. Secchi ${ }^{(3)}$, em seu estudo realizado, afirma que, apesar de apresentarem uma auto percepção corporal e um índice de massa corporal (IMC) considerados normais, encontravam-se insatisfeitos com sua aparência física.

Através da prática do exercício físico, focando a resistência cardiorrespiratória, obtém-se em alguns indivíduos a busca incessante por um corpo massérrimo, outros se encontram com compunções alimentares e ao se arrependerem, passam horas na academia. Estas duas práticas são consideradas distúrbios da IC que atingem, na maioria das vezes as mulheres. No caso dos homens, acontece a busca pelo aumento do volume dos músculos, através do componente da aptidão física, a força, muitos deles extrapolam a carga resistida, das séries de exercícios e da duração destes, em busca de um corpo "Hipertrofiado"(5).

Estudos demonstram que em estudantes da área da saúde, como Educação Física, devido a um interesse pessoal pelas questões corporais, a insatis- fação com a IC tende a ser maior, em comparação aos acadêmicos de outras áreas $^{(6)}$.

Neste sentido, o estilo de vida, passa a definir a saúde do indivíduo, pois se configura como conjunto de ações diárias do ser humano, refletindo em seus valores, e oportunidades pessoais, que vão da alimentação até suas escolhas profissionais e pessoais ${ }^{(7)}$.

Portanto, diante do exposto, o presente estudo teve o objetivo de comparar a Percepção da IC Atual e Percepção da IC que se Pretende Ter com o IMC, Percentual de Gordura Corporal e Prática de Atividade Física de estudantes de Educação Física.

\section{MÉTODOS}

Este é um estudo quanti-quantitativo, com delineamento descritivo e transversal. A pesquisa foi realizada com os estudantes de Educação Física de Porto Velho-RO. Este trabalho foi aprovado pelo Comitê de Ética em Pesquisa da Universidade Federal de Rondônia seguindo as diretrizes metodológicas de acordo com a declaração de Helsinque ${ }^{(8)}$.

\section{Participantes}

Participaram estudantes de Educação Física de ambos os gêneros. O critério de seleção foi por conveniência, totalizando 43 alunos. O período determinado para coleta foi de 10 dias, iniciando dia 16 e terminando no dia 25 de setembro de 2019. Como critério de inclusão: ser aluno do curso de Educação Física e estar de acordo com o termo de consentimento livre e esclarecido (TCLE). Como critério de exclusão: alunos com idade inferior a 18 anos ou com idade superior a 60 anos.

\section{Desenho Experimental}

O pesquisador abordou cada sujeito individualmente, para a apresentação dos procedimentos e propósitos da pesquisa, ao aceitarem, os participantes assinaram o TCLE. Em seguida foram avaliados com os instrumentos da pesquisa que são baseados em questionários.

Inicialmente, foram coletados dados relevantes à pesquisa, incluindo, aspectos de identificação. Em seguida, foram utilizados o instrumento para avaliação da IC, sendo este composto de nove silhuetas, onde o participante precisa identificar o formato de corpo que ele acredita ter e qual gostaria de $\operatorname{ter}^{(9)}$. Após essa etapa, foram coletados dobras cutâneas e dados antropométricos como massa $(\mathrm{Kg})$ e estatura (m), também servindo para cálculo de Índice de Massa Corporal ${ }^{(10,11)}$. Por fim, aplicou-se o Questionário Internacional de Atividade Física (IPAQ), versão curta ${ }^{(12,13)}$. 
A percepção corporal foi avaliada comparando a figura da IC atual com os dados de Índice de Massa Corporal (IMC). E a satisfação corporal foi avaliada comparando a figura da IC que gostaria de ter com a IC atual, sendo avaliada a diferença entre a silhueta atual e a desejada.

\section{Protocolo de Avaliação da IC}

Para investigar a percepção da IC foi aplicado o instrumento proposto por Stunkard ${ }^{(9)}$, composto de nove silhuetas (ver Figura 1). A partir da apresentação do conjunto de silhuetas, foram realizadas duas perguntas aos acadêmicos: I - Qual o número da silhueta que você considera mais semelhante com sua aparência real? II - E qual o número da silhueta que você gostaria de ter?

A classificação das silhuetas segue a seguinte recomendação: Magreza severa (1), Magreza (2 e 3),
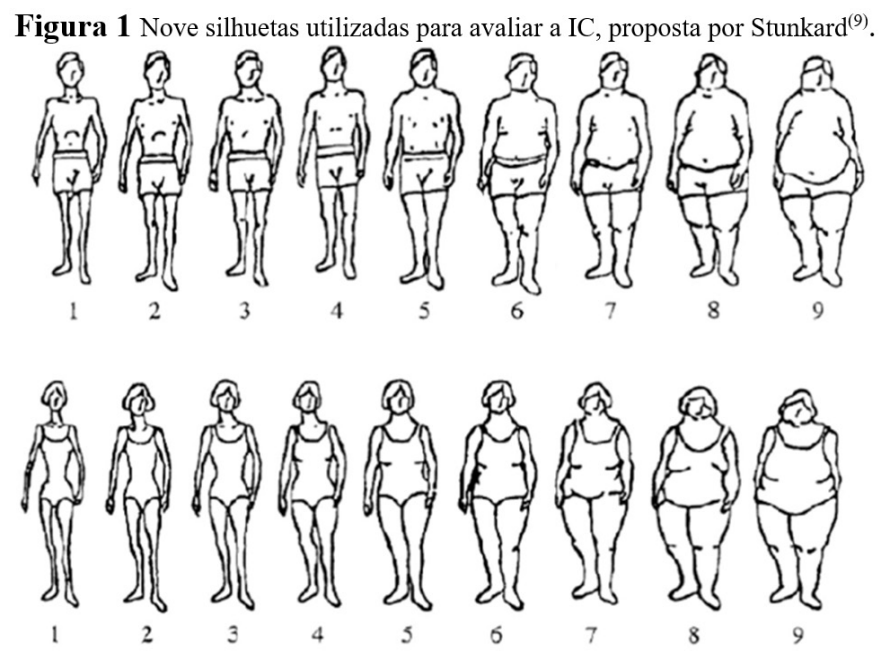

Fonte: Stunkard, $1983^{(9)}$

Eutrofia (4 e 5), sobrepeso (6 e 7) e Obesidade (8 e $9)$, onde a primeira linha é designada ao corpo atual e a segunda linha para o corpo que gostaria de ter ${ }^{(14)}$.

Para a análise dos dados, após escolha da figura que mais se pareceu com o seu corpo e a figura que ele gostaria de se parecer, os participantes foram classificados em: GG - Os que gostariam de ganhar peso (quando a silhueta real for mais magra que a desejada); GS - Os que estiveram satisfeitos com o peso (quando as silhuetas desejadas forem às mesmas); GP - Aqueles que gostariam de perder peso (quando a silhueta real for mais obesa que a desejada) ${ }^{(14)}$.

\section{Protocolo de Avaliação Antropométrica}

A espessura das dobras cutâneas, seguiram a técnica preconizada por Heyward e Stolarczyk ${ }^{(11)}$. Foram coletadas as dobras Subescapular (DSE), tríceps (DCT), bíceps (DCB) e supra ilíaca (DSI) utilizando-se um aidpômetro. O percentual de gordura foi obtido a partir da comparação com os valores de referência que representam risco de doenças e distúrbios associados à desnutrição (RDAO), abaixo da média $(A B M)$, média $(M)$, acima da média ( $A C M)$ e risco de doenças associadas à obesidade (RADO), apresentados na tabela $1 \mathrm{com}$ o da soma das quatros dobras cutâneas realizada pelo cálculo:

\section{$\sum=\operatorname{DCT}(\mathbf{m m})+\mathrm{DCB}(\mathbf{m m})+\mathrm{DSE}(\mathbf{m m})+\mathrm{DSI}(\mathbf{m m})$}

Tabela 1 Valores de referência de percentual de gordura corporal.

\begin{tabular}{lll}
\hline Valores de referência & para & \multicolumn{2}{l}{ Gordura Corpórea (\%) } \\
\cline { 3 - 3 } porcentuais de gordura corpórea: & Homens & Mulheres \\
\hline RDAO & $\leq 5$ & $\leq 8$ \\
ABM & 6 a 14 & 9 a 22 \\
M & 15 & 23 \\
ACM & 15 a 24 & 24 a 31 \\
RADO & $\geq 25$ & $\geq 32$ \\
\hline
\end{tabular}

Fonte : Lohman et al, 2005(15).

Para obtenção dos dados acerca do IMC, foi utilizada uma balança digital eletrônica, com capacidade de até $150 \mathrm{~kg}$, marca Plenna e um estadiômetro de madeira com precisão de $0,1 \mathrm{~cm}$, fixado verticalmente em uma parece lisa e sem rodapé. A avaliação de massa corporal e estatura foram seguidas de acordo com as recomendações de Heyward e Sto$\operatorname{larczyk}^{(11)}$. A classificação do IMC foi feita de acordo com a Organização Mundial de Saúde ${ }^{(16)}$.

\section{Protocolo de avaliação IPAQ}

O nível de atividade física foi determinado por meio do Questionário Internacional de Atividade Física (IPAQ) versão curta ${ }^{(13)}$. Este método de avaliação do nível de atividade física possui 4 questões subdividas em itens "a" e "b", considerando os critérios de frequência, duração e tipo de atividade física. Os participantes podem ser classificados em sedentários, insuficientemente ativo, ativo e muito ativo. São considerados fisicamente ativos os indivíduos que realizarem ao menos 150 minutos de atividade física semanal por cinco ou mais dias da semana e sedentários os que realizarem menos de 10 minutos diários de atividade física. Os indivíduos que relatarem realizar atividade física, mas não alcançarem as recomendações propostas, são considerados insuficientemente ativos.

\section{Análise Estatística}

Os dados coletados foram catalogados através de fichas protocolares e posteriormente tabulados no programa Microsoft Office Excel $2010^{\oplus}$, para verifi- 
cação das médias, desvios padrão e percentuais, em uma análise de estatística descritiva.

\section{RESULTADOS E DISCUSSÃO}

No presente estudo participaram da pesquisa 43 estudantes de Educação Física do $2^{\circ}, 4^{\circ}, 6^{\circ}$ e $8^{\circ}$ período, com idades entre 18 a 39 anos. Destes estudantes que participaram, 23 alunos $(53,5 \%)$ são do sexo masculino e 20 estudantes (46,5\%), são do sexo feminino (Tabela 2a).

Conforme os resultados obtidos em relação à Distribuição da Classificação do IMC (tabela 2b), observa-se que do total de alunos avaliados o predominante foi Eutrofia com 62,7\%. Apesar da maioria dos estarem eutróficos, os estudantes de educação física apresentavam-se com sobrepeso $(16,3 \%)$ ou obesidade (18,6\%).

Ao comparar a classificação do IMC com o percentual de gordura (tabela $2 \mathrm{c}$ ) verifica-se que 11,7\% dos estudantes eutróficos estavam com o percentual de gordura abaixo da média, ou seja, apresentavam na composição corporal maior quantidade de massa magra em relação à massa gorda. Apesar da maioria dos estudantes estarem eutróficos, 34,9\% dos estudantes apresentavam-se com sobrepeso ou obesidade através da classificação de IMC/gordura, os quais apresentaram porcentagem de gordura classificados acima da média e com risco de doenças associadas à obesidade, ou seja, apresentam maior quantidade de massa gorda em relação a massa magra.

Enquanto que $27,9 \%$ dos estudantes estavam eutróficos pelo IMC, porém apresentaram com percentual de gordura acima da média, mostrando que o IMC não deve ser utilizado unicamente para avaliar o estado nutricional do indivíduo, para conseguir assim reverter os riscos de possíveis patologias e hábitos. Apesar disso, 23,2\% dos eutróficos que estão com o percentual de gordura classificados na média, portanto, estão dentro dos padrões de normalidade adotados como referência.

Percebeu-se que os alunos com sobrepeso ou obesidade $(34,9 \%)$, pelo IMC/gordura (tabela $2 \mathrm{c}$ ), não se percebem com esta IC, conforme a Distribuição da IC Atual (Tabela 2d), pois apenas $11,6 \%$ se

Tabela 2 Distribuição de Gênero, da IC, da IC que gostaria de ter e do nível de atividade física; distribuição da classificação de IMC e IMC/Gordura.

\begin{tabular}{|c|c|c|}
\hline a. Distribuição de Gênero & $\mathbf{N}$ & $\%$ \\
\hline Feminino & 20 & 46,50 \\
\hline Masculino & 23 & 53,50 \\
\hline b. Distribuição da Classificação do IMC & $\mathbf{N}$ & $\%$ \\
\hline Eutrofia & 27 & 62,70 \\
\hline Magreza & 1 & 2,40 \\
\hline Obesidade & 8 & 18,60 \\
\hline Sobrepeso & 7 & 16,30 \\
\hline Distribuição da Classificação de IMC/Gordura & $\mathbf{N}$ & $\%$ \\
\hline Eutrofia $/ \mathrm{ABM}$ & 5 & 11,70 \\
\hline Eutrofia / ACM & 12 & 27,90 \\
\hline Eutrofia / M & 10 & 23,20 \\
\hline Magreza / M & 1 & 2,30 \\
\hline Obesidade / ACM & 3 & 6,90 \\
\hline Obesidade / RADO & 5 & 11,70 \\
\hline Sobrepeso / ACM & 5 & 11,70 \\
\hline Sobrepeso / RDAO & 2 & 4,60 \\
\hline d. Distribuição da IC Atual & $\mathbf{N}$ & $\%$ \\
\hline Eutrofia & 9 & 20,90 \\
\hline Magreza & 26 & 60,50 \\
\hline Magreza severa & 3 & 7,00 \\
\hline Sobrepeso & 5 & 11,60 \\
\hline e. Distribuição da IC que Gostaria de Ter & $\mathbf{N}$ & $\%$ \\
\hline Eutrofia & 11 & 25,5 \\
\hline Magreza & 28 & 65,1 \\
\hline Magreza severa & 4 & 9,4 \\
\hline Sobrepeso & 0 & 0 \\
\hline f. Distribuição do Nível de Atividade Física & $\mathbf{N}$ & $\%$ \\
\hline Ativo & 10 & 23,30 \\
\hline Muito Ativo & 17 & 39,50 \\
\hline Insuficientemente Ativa & 15 & 34,90 \\
\hline Sedentário & 1 & 2,30 \\
\hline
\end{tabular}


veem com sobrepeso, o que pode não ser benéfico, pois estes podem não se importaram com a realização de atividades física e com a prática de consumo de uma dieta saudável e equilibrada para melhora do seu estado nutricional.

Quando observado os dados da percepção da IC atual (tabela 2d), 67,5\% dos estudantes de Educação Física se percebem com algum grau de magreza, verificando, assim, uma possível distorção da IC, pois o método do IMC/gordura obteve-se apenas 2,3\% de estudantes com magreza.

A insatisfação pela magreza poder ser recorrente devido a fatores como o padrão de corpo ideal e o meio de atuação profissional. No estudo de Rech et $\mathrm{al}^{(17)}$, o qual pesquisou sobre a percepção da IC em estudantes do curso de Educação Física, obteve-se em relação à IC atual dos estudantes, 56,6\% (Homens) e $74,1 \%$ (Mulheres) percebem-se classificados com algum grau de magreza, havendo distorção na IC, pois, pelo IMC, obteve-se apenas $2,4 \%$ de estudantes com magreza, corroborando com os dados desta pesquisa.

Os resultados demonstram que os estudantes de Educação Física estão com as suas percepções de IC atual distante da realidade, podendo ser, assim, uma consequência da influência da mídia, sociedade e meio esportivo ao idealizar que corpos perfeitos desse tipo de profissional da área da saúde devem ser extremamente fortes e musculosos.

Observa-se que os estudantes da amostra tendem a querer uma IC atual (tabela 2e) de magreza $(65,1 \%)$ e de magreza severa $(9,4 \%)$, representando uma maior parcela em relação a eutrofia $(25,5 \%)$; nenhum participante do estudo relatou desejar ter uma IC com sobrepeso.

Segundo O'brien et $\mathrm{al}^{(18)}$, o fato dessa percepção ser advinda dos acadêmicos dos cursos de Educação Física é interessante, uma vez que serão os mesmos que atuarão com as práticas corporais no âmbito escolar e não escolar. Em estudos prévios já se havia relatado que acadêmicos do curso de Educação Física apresentam uma preocupação maior com a aparência física, quando comparada com acadêmicos de outros cursos.

Os resultados do nível de atividade física (tabela 2f) apontaram que 39,5\% são Muito Ativos, ou seja, são os indivíduos que realizam atividades vigorosas $5 \mathrm{x}$ por semana por pelo menos 30 minutos ou 3 dias /semana e 20 minutos de forma vigorosa + Moderada e/ou Caminhada: 5 dias/semana de $30 \mathrm{mi}-$ nutos. Esse resultado pode estar relacionado ao perfil dos participantes, pois, no curso de Educação Física, a prática de Atividade física é constante no dia-a-dia do indivíduo, onde 34,9\% dos indivíduos apresentavam-se insuficientemente ativos, que são aqueles que realizam por pelo menos 10 minutos contínuos atividade na semana. A prevalência de Sedentários, foi de apenas $2,3 \%$.

Os resultados do presente estudo são semelhantes ao estudo de Bosi et $\mathrm{al}^{(19)}$, onde a maioria dos universitários foram classificados como eutróficos e, mesmo no grupo com estado nutricional normal, observou-se uma grande insatisfação com a IC, os quais gostariam de ser magros. Essas divergências entre o estado nutricional e a insatisfação com a IC, podem estar relacionadas a uma preocupação exagerada com a estética corporal culturalmente imposta, fazendo com que o padrão de beleza idealizado não corresponda aos padrões considerados adequados para a saúde, o que poderia ser trabalhado por meio de medidas de educação em saúde.

Já as alunas podem fazer dieta extremamente rígida e se abster de alimentos com uma busca desenfreada pela magreza, distúrbio causado por uma distorção da $\mathrm{IC}^{(19)}$.

No estudo de Coqueiro et $\mathrm{a}^{(20)}$, foi verificado que $83,2 \%$ dos indivíduos foram classificados como eutróficos segundo o IMC, e pela escala de silhueta a maioria dos indivíduos $(78,8 \%)$ estavam insatisfeitos com a própria IC. Além disso, observou-se que 49,2\% dos indivíduos apresentaram desejo de reduzir o tamanho da silhueta, enquanto que $26,6 \%$ desejavam aumentar, diferentemente deste estudo. Os referidos dados são, em parte, corroborados por este estudo.

A tabela 3 apresenta a relação da classificação do IMC com a IC Atual; Comparação da IC atual com a IC desejada do participante; Comparação da IC atual com o Nível de prática de atividade física.

Com relação à comparação do IMC com a IC que gostariam de ter (tabela $3 \mathrm{~b}$, observa-se uma discrepância nos dados, onde, pelo IMC (tabela $2 b$ ), a maioria está adequado $(62,7 \%)$, e pela escala de silhueta (tabela 2e) os estudantes gostariam de ser magros $(74,5 \%)$, podendo gerar comportamentos que levam a ocorrência de práticas inadequadas de dietas e de exercícios físicos.

Percebe-se que existe uma insatisfação com a IC, assim como uma distorção da autopercepção corporal com a condição do estado nutricional. Existem evidências que indicam que a mídia promove distúrbios da IC e alimentar, influenciando a crescente insatisfação das pessoas com a própria aparência ${ }^{(21)}$.

A IC é uma construção multidimensional que descreve as representações internas da estrutura corporal e da aparência física de si e dos outros, ou seja, é a ilustração ou figura que se tem na mente do ta- 
Tabela 3 Comparação entre IC atual e classificação de IMC, entre IC e IC que gostaria de ter e entre IC atual e nível de atividade física em valores percentuais.

\begin{tabular}{|c|c|c|c|c|c|c|c|c|c|c|}
\hline \multirow{2}{*}{$\begin{array}{cc}\text { a. } & \text { IC atual } \\
\text { Classificação IMC }\end{array}$} & \multicolumn{2}{|c|}{ Eutrofia } & \multicolumn{2}{|c|}{ Sobrepeso } & \multicolumn{2}{|c|}{ Obesidade } & \multicolumn{2}{|c|}{ Magreza } & \multicolumn{2}{|c|}{ Total } \\
\hline & $\mathbf{N}$ & $\%$ & $\mathbf{N}$ & $\%$ & $\mathbf{N}$ & $\%$ & $\mathbf{N}$ & $\%$ & $\mathbf{N}$ & $\%$ \\
\hline Eutrofia & 4 & $15 \%$ & 3 & $43 \%$ & 2 & $25 \%$ & 0 & 0 & 9 & $21 \%$ \\
\hline Magreza & 20 & $77 \%$ & 1 & $14 \%$ & 4 & $50 \%$ & 1 & $50 \%$ & 26 & $60 \%$ \\
\hline Magreza severa & 2 & $8 \%$ & 0 & 0 & 0 & 0 & 1 & $50 \%$ & 3 & $7 \%$ \\
\hline Sobrepeso & 0 & 0 & 3 & $43 \%$ & 2 & $25 \%$ & 0 & 0 & 5 & $12 \%$ \\
\hline Total & 26 & $60 \%$ & 7 & $16 \%$ & 8 & $19 \%$ & 2 & $5 \%$ & 43 & $100 \%$ \\
\hline \multirow[t]{2}{*}{ b. } & \multicolumn{2}{|c|}{ Eutrofia } & \multicolumn{2}{|c|}{ Sobrepeso } & \multicolumn{2}{|c|}{ Obesidade } & \multicolumn{2}{|c|}{ Magreza } & \multicolumn{2}{|c|}{ Total } \\
\hline & $\mathbf{N}$ & $\%$ & $\mathbf{N}$ & $\%$ & $\mathbf{N}$ & $\%$ & $\mathbf{N}$ & $\%$ & $\mathbf{N}$ & $\%$ \\
\hline Eutrofia & 3 & $33 \%$ & 3 & $11 \%$ & 1 & $33 \%$ & 4 & $80 \%$ & 11 & $26 \%$ \\
\hline Magreza & 4 & $44 \%$ & 21 & $81 \%$ & 2 & $67 \%$ & 1 & $20 \%$ & 28 & $65 \%$ \\
\hline Magreza severa & 2 & $23 \%$ & 2 & $8 \%$ & 0 & 0 & 0 & 0 & 4 & $9 \%$ \\
\hline Sobrepeso & 0 & 0 & 0 & $0 \%$ & 0 & 0 & 0 & 0 & 0 & 0 \\
\hline Total & 9 & $21 \%$ & 26 & $60 \%$ & 3 & $7 \%$ & 5 & $12 \%$ & 43 & $100 \%$ \\
\hline \multirow{2}{*}{$\begin{array}{cc}\text { c. } & \text { IC Atual } \\
\text { Nível de atividade física }\end{array}$} & \multicolumn{2}{|c|}{ Ativo } & \multicolumn{2}{|c|}{ Muito Ativo } & \multicolumn{2}{|c|}{ Insuficientemente ativa } & \multicolumn{2}{|c|}{ Sedentário } & \multicolumn{2}{|c|}{ Total } \\
\hline & $\mathbf{N}$ & $\%$ & $\mathbf{N}$ & $\%$ & $\mathbf{N}$ & $\%$ & $\mathbf{N}$ & $\%$ & $\mathbf{N}$ & $\%$ \\
\hline Eutrofia & 4 & $40 \%$ & 1 & $6 \%$ & 3 & $20 \%$ & 1 & $100 \%$ & 9 & $21 \%$ \\
\hline Magreza & 5 & $50 \%$ & 11 & $65 \%$ & 10 & $66 \%$ & 0 & 0 & 26 & $60 \%$ \\
\hline Magreza severa & 1 & $10 \%$ & 1 & $6 \%$ & 1 & $7 \%$ & 0 & 0 & 3 & $7 \%$ \\
\hline Sobrepeso & 0 & 0 & 4 & $23 \%$ & 1 & $7 \%$ & 0 & 0 & 5 & $12 \%$ \\
\hline Total & 10 & $23 \%$ & 17 & $40 \%$ & 15 & $35 \%$ & 1 & $2 \%$ & 43 & $100 \%$ \\
\hline
\end{tabular}

Classificação do IMC, IC Atual e do Nível de prática de atividade física. Fonte: Próprio Autor.

manho, IC e forma do corpo. Pessoas com distúrbios na percepção da IC superestimam em maior grau a sua $\mathrm{IC}^{(22)}$. Análises têm estabelecido que os estudantes universitários e, em especial aqueles dos cursos de Educação Física, merecem uma particular atenção com relação ao tema IC.

Ao escolherem esta profissão os acadêmicos veem-se associados a uma imagem de corpos esculturais e hábitos alimentares saudáveis o que pode levar índices ainda maiores de insatisfação. No estudo de Damasceno et $\mathrm{al}^{(1)}$, não somente as mulheres buscam o corpo perfeito (mais magro e menos volumoso), mas também os homens querem ter um corpo mais forte e volumoso.

Porém, o excesso de carga extracurricular, devido à abrangência da área verificado neste grupo, dentre outros fatores, acaba por favorecer a adoção de hábitos não saudáveis de controle do peso (indução de vômitos, exercícios físicos rigorosos, dietas drásticas, uso de suplementos sem prescrição de um profissional habilitado) no decorrer da formação ${ }^{(23)}$.

A mídia e o imaginário coletivo parecem estabelecer uma estreita relação entre a forma do corpo e a saúde, como se todos os regimes, dietas, exercícios físicos pudessem ser utilizados no sentido do indivíduo cuidar-se melhor, tornando-se mais saudável. No entanto, na última década, os casos de transtornos alimentares proliferaram principalmente os quadros de bulimia, anorexia nervosa, vigorexia e obesidade, criando-se ambulatórios de saúde específicos para o tratamento médico, nutricional e psicológico ${ }^{(24)}$.

A tabela 4, apresenta a relação de classificação IMC e IC atual dos gêneros feminino e masculino dos estudantes de forma distinta.

Tabela 4 Comparação do IMC com a Percepção da IC Atual separada por Gênero em valores percentuais.

\begin{tabular}{|c|c|c|c|c|c|c|c|c|c|c|}
\hline \multirow{2}{*}{$\begin{array}{c}\text { IC Atual } \\
\text { IMC (FEMININO) }\end{array}$} & \multicolumn{2}{|c|}{ Eutrofia } & \multicolumn{2}{|c|}{ Sobrepeso } & \multicolumn{2}{|c|}{ Obesidade } & \multicolumn{2}{|c|}{ Magreza } & \multicolumn{2}{|c|}{ Total } \\
\hline & $\mathbf{N}$ & $\%$ & $\mathbf{N}$ & $\%$ & $\mathbf{N}$ & $\%$ & $\mathbf{N}$ & $\%$ & $\mathbf{N}$ & $\%$ \\
\hline Eutrofia & 2 & $50 \%$ & 11 & $73 \%$ & 1 & $100 \%$ & 0 & 0 & 14 & $70 \%$ \\
\hline Sobrepeso & 0 & 0 & 1 & $7 \%$ & 0 & 0 & 0 & 0 & 1 & $5 \%$ \\
\hline Obesidade & 2 & $50 \%$ & 2 & $13 \%$ & 0 & 0 & 0 & 0 & 4 & $20 \%$ \\
\hline Magreza & 0 & 0 & 1 & $7 \%$ & 0 & 0 & 0 & 0 & 1 & $5 \%$ \\
\hline Total & 4 & $20 \%$ & 15 & $75 \%$ & 1 & $5 \%$ & 0 & 0 & 20 & $100 \%$ \\
\hline IC Atual & \multicolumn{2}{|c|}{ Eutrofia } & \multicolumn{2}{|c|}{ Sobrepeso } & \multicolumn{2}{|c|}{ Obesidade } & \multicolumn{2}{|c|}{ Magreza } & \multicolumn{2}{|c|}{ Total } \\
\hline IMC (MASCULINO) & $\mathbf{N}$ & $\%$ & $\mathbf{N}$ & $\%$ & $\mathbf{N}$ & $\%$ & $\mathbf{N}$ & $\%$ & $\mathbf{N}$ & $\%$ \\
\hline Eutrofia & 2 & $40 \%$ & 9 & $82 \%$ & 1 & $50 \%$ & 0 & $0 \%$ & 12 & $52 \%$ \\
\hline Sobrepeso & 3 & $60 \%$ & 0 & $0 \%$ & 0 & 0 & 3 & $60 \%$ & 6 & $26 \%$ \\
\hline Obesidade & 0 & $0 \%$ & 2 & $18 \%$ & 0 & 0 & 2 & $40 \%$ & 4 & $17 \%$ \\
\hline Magreza & 0 & $0 \%$ & 0 & $0 \%$ & 1 & $50 \%$ & 0 & 0 & 1 & $5 \%$ \\
\hline Total & 5 & $21 \%$ & 11 & $49 \%$ & 2 & $9 \%$ & 5 & $21 \%$ & 23 & $100 \%$ \\
\hline
\end{tabular}

Fonte: Próprio Autor. 
Importante reforçar que quando analisado pelo gênero observa-se que $73 \%$ das alunas com o IMC Eutrófico percebem-se com sobrepeso e 50\% das alunas obesas pelo IMC, percebem-se eutróficas. Em relação ao gênero masculino, verifica-se que $82 \%$ dos alunos Eutróficos em relação ao IMC, percebem-se com sobrepeso. Enquanto que $60 \%$ dos alunos com Sobrepeso no IMC percebem-se eutróficos.

O estudo de Silva et al ${ }^{(23)}$, corrobora a presente investigação. $\mathrm{O}$ autor verificou que a prevalência de insatisfação pela magreza foi 2,71 vezes maior no sexo masculino. A maior parte dos acadêmicos insatisfeitos pela magreza no seu estudo foram classificados como eutróficos $(29,8 \%)$, e na análise considerando o estado nutricional, foi observada insatisfação pela magreza nos acadêmicos 4,51 vezes maior do que os acadêmicos classificados com sobrepeso/obesidade.

Com relação à IC que gostaria de ter (desejada), com o IMC/\%Gordura (Tabela 5) os estudantes ACM e RDAO Eutróficos (27,9\%) tendem a ter IC desejada de magreza (36\%) e magreza severa (50\%). Já aqueles com o IMC/gordura ACM e RDAO $(34,9 \%)$ tendem a ter IC desejada de Eutrofia (54\%) e de magreza (26\%).

Percebe-se uma insatisfação pelos estudantes em relação à composição corporal, uma vez que tanto os acadêmicos eutróficos ACM e RDAO (27,9\%) quanto os que estão com excesso de peso $(34,9 \%)$ apresentam maior quantidade de massa gorda.

Segundo Coqueiro et $\mathrm{al}^{(20)}$, um dado impor- tante encontrado foi a diferença significativa entre os dois indicadores (IMC e dobras cutâneas), na classificação do estado nutricional. Os resultados mostraram que o IMC classificou a maioria dos universitários como eutróficos, enquanto que o percentual de gordura indicou que mais da metade destes encontravam-se em estado de inadequação nutricional, confirmando que, independente do sexo, as dobras cutâneas são melhores preditores da insatisfação com a IC em universitários, enquanto que o IMC parece não ser um determinante significativo da percepção da IC. Esta pesquisa está de acordo com os dados da presente investigação.

Em seguida, apresenta-se a tabela 5, que revela a relação IMC/\%G, IC desejada e Nível de atividade física.

Alguns autores apontam que os níveis de atividade física variam entre os diversos cursos e que os alunos do curso de Educação Física, geralmente, são mais ativos. A sociedade acredita que estes alunos apresentam hábitos mais saudáveis em seu cotidiano, sobretudo, por terem disciplinas curriculares que tratam dos conceitos de Educação em Saúde e Promoção da Saúde. No entanto, os resultados referentes à adoção de um estilo de vida ativo nesta população são controversos. Resende et $\mathrm{al}^{(25)}$, evidenciam que apenas o conhecimento acerca dos fatores de risco não é suficiente para que as práticas saudáveis de estilo de vida sejam adotadas.

Tabela 5 Relação entre IC Desejada e IMC/\%G e entre Nível de Atividade física e IMC/\%G.

\begin{tabular}{|c|c|c|c|c|c|c|c|c|c|c|}
\hline \multicolumn{11}{|c|}{ IC Desejada } \\
\hline \multirow{2}{*}{ IMC / \% } & \multicolumn{2}{|c|}{ Eutrofia } & \multicolumn{2}{|c|}{ Sobrepeso } & \multicolumn{2}{|c|}{ Obesidade } & \multicolumn{2}{|c|}{ Magreza } & \multicolumn{2}{|c|}{ Total } \\
\hline & $\mathbf{N}$ & $\%$ & $\mathbf{N}$ & $\%$ & $\mathbf{N}$ & $\%$ & $\mathbf{N}$ & $\%$ & $\mathbf{N}$ & $\%$ \\
\hline Eutrofia /ABM & 4 & $37 \%$ & 1 & $3 \%$ & 0 & $0 \%$ & 0 & $0 \%$ & 5 & $12 \%$ \\
\hline Eutrofia / ACM & 0 & $0 \%$ & 10 & $36 \%$ & 2 & $50 \%$ & 0 & $0 \%$ & 12 & $28 \%$ \\
\hline Eutrofia / M & 1 & $9 \%$ & 9 & $32 \%$ & 0 & $0 \%$ & 0 & $0 \%$ & 10 & $23 \%$ \\
\hline Magreza / M & 0 & $0 \%$ & 1 & $3 \%$ & 0 & $0 \%$ & 0 & $0 \%$ & 1 & $2 \%$ \\
\hline Obesidade / ACM & 0 & $0 \%$ & 3 & $12 \%$ & 0 & $0 \%$ & 0 & $0 \%$ & 3 & $7 \%$ \\
\hline Obesidade / RADO & 2 & $18 \%$ & 2 & $8 \%$ & 1 & $25 \%$ & 0 & $0 \%$ & 5 & $12 \%$ \\
\hline Sobrepeso / ACM & 4 & $36 \%$ & 1 & $3 \%$ & 0 & $0 \%$ & 0 & $0 \%$ & 5 & $12 \%$ \\
\hline Sobrepeso / RDAO & 0 & $0 \%$ & 1 & $3 \%$ & 1 & $25 \%$ & 0 & $0 \%$ & 2 & $4 \%$ \\
\hline \multirow[t]{2}{*}{ Total } & 11 & $25 \%$ & 28 & $65 \%$ & 4 & $10 \%$ & 0 & $0 \%$ & 43 & $100 \%$ \\
\hline & \multicolumn{6}{|c|}{ Nível de Atividade Física } & & & & \\
\hline \multirow{2}{*}{ IMC / \% } & \multicolumn{2}{|c|}{ Ativo } & \multicolumn{2}{|c|}{ Muito Ativo } & \multicolumn{2}{|c|}{ Insuficientemente ativa } & \multicolumn{2}{|c|}{ Sedentário } & \multicolumn{2}{|c|}{ Total } \\
\hline & $\mathbf{N}$ & $\%$ & $\mathbf{N}$ & $\%$ & $\mathbf{N}$ & $\%$ & $\mathbf{N}$ & $\%$ & $\mathbf{N}$ & $\%$ \\
\hline Eutrofia /ABM & 1 & $10 \%$ & 4 & $23 \%$ & 0 & $0 \%$ & 0 & $0 \%$ & 5 & $12 \%$ \\
\hline Eutrofia / ACM & 2 & $20 \%$ & 3 & $18 \%$ & 7 & $46 \%$ & 0 & $0 \%$ & 12 & $28 \%$ \\
\hline Eutrofia / M & 3 & $30 \%$ & 3 & $18 \%$ & 4 & $27 \%$ & 0 & $0 \%$ & 10 & $23 \%$ \\
\hline Magreza / M & 0 & 0 & 0 & 0 & 1 & $7 \%$ & 0 & $0 \%$ & 1 & $2 \%$ \\
\hline Obesidade / ACM & 1 & $10 \%$ & 2 & $12 \%$ & 0 & $0 \%$ & 0 & $0 \%$ & 3 & $7 \%$ \\
\hline Obesidade / RADO & 1 & $10 \%$ & 1 & $5 \%$ & 2 & $13 \%$ & 1 & $100 \%$ & 5 & $12 \%$ \\
\hline Sobrepeso / ACM & 2 & $20 \%$ & 2 & $12 \%$ & 1 & $7 \%$ & 0 & $0 \%$ & 5 & $12 \%$ \\
\hline Sobrepeso / RDAO & 0 & 0 & 2 & $12 \%$ & 0 & $0 \%$ & 0 & $0 \%$ & 2 & $4 \%$ \\
\hline Total & 10 & $23 \%$ & 17 & $39 \%$ & 15 & $35 \%$ & 1 & $3 \%$ & 43 & $100 \%$ \\
\hline
\end{tabular}

Fonte: Próprio Autor. 


\section{CONCLUSÃO}

Os participantes que fizeram parte da amostra apresentaram divergências entre a percepção da IC atual, com o IMC e o percentual de gordura. Tais divergências corroboram com estudos anteriores realizados com estudantes de educação física. A percepção da IC atual e a IC pretendida remetem a possíveis influências, envolvendo cobranças sociais relacionadas a ideia de "corpo perfeito" e "saúde". Estudantes de educação física possuem um comportamento relativamente ativo devido suas rotinas de atividades no curso ou atividades estimuladas pelo convívio com a atividade física, mesmo sendo encontrado na literatura pontos controversos, nesse estudo foi encontrado um valor baixo de participantes sedentários.

\section{REFERÊNCIAS}

1. Damasceno VO, Vianna VR, Vianna JM, Lacio M, Lima JRP, Novaes JS. Imagem corporal e corpo ideal. Revista brasileira de ciência e movimento. 2008;14(2):81-94.

2. Almeida GANd, Santos JEd, Pasian SR, Loureiro SR. Percepçao de tamanho e forma coporal de mulheres: Estudo exploratório. Psicologia em estudo. 2005;10(1):27-35.

3. Secchi K, Camargo BV, Bertoldo RB. Body image perception and body's social representations/Percepcao da imagem corporal e representacoes sociais do corpo. Psicologia: teoria e pesquisa. 2009;25(2):229-37.

4. McCabe MP, Ricciardelli LA. Sociocultural influences on body image and body changes among adolescent boys and girls. The Journal of social psychology. 2003;143(1):5-26.

5. Koblitz A, Contini CS, Pich S. Aptidão Física Promovendo a Construção de um Ideal de Imagem Corporal Saudável.

6. Ferrari EP, Silva DAS, Petroski EL. Associação entre percepção da imagem corporal e estágios de mudança de comportamento em acadêmicos de educação física. Rev bras cineantropom desempenho hum. 2012;14(5):535-44.

7. Oliveira HF, Vieira FS, dos Santos Leal KA, Novelli C, Noda DKG, Risso HR, et al. Estresse e qualidade de vida de estudantes universitários. Revista CPAQV-Centro de Pesquisas Avançadas em Qualidade de Vida-CPAQV Journal. 2015;7(2).

8. Associação Médica Mundial. Declaração de Helsinque. Princípios éticos para a pesquisa em seres humanos Helsinque. 1964.

9. Stunkard AJ. Use of the Danish Adoption Register for the study of obesity and thinness. Res Publ Assoc Res Nerv Ment Dis. 1983;60:115-20.

10. Fontoura ASd, Formentin CM, Abech EA. Guia prático de avaliação física: uma abordagem didática, abrangente e atualizada: Phorte Editora LTDA; 2011.

11. Heyward VH, Stolarczyk LM. Avaliação da composição corporal aplicada2000.

12. Miranda F. Como utilizar o questionário Internacional de Actividade Física IPAQ Versão Curta In https://www.youtube.com/watch?time_continue $=4 \& v=T Z j-y s-v 7 P E$. Acedido em. $2016 ; 7$.

13. Vespasiano BdS, Dias R, Correa DA. A utilização do questionário internacional de atividade física (IPAQ) como ferramenta diagnóstica do nível de aptidão física: uma revisão no Brasil. Saúde em Revista. 2012;12(32):49-54.
14. Fernandes AER. Avaliação da imagem corporal, hábitos de vida e alimentares em crianças e adolescentes de escolas públicas e particulares de Belo Horizonte. Belo Horizonte (MG): UFMG. 2007.

15. Lohman T, Wang Z, Going SB. Human body composition: Human Kinetics; 2005.

16. World Health Organization. Diet, nutrition, and the prevention of chronic diseases: report of a joint WHO/FAO expert consultation: World Health Organization; 2003.

17. Rech CR, Araújo EDdS, Vanat JDR. Autopercepção da imagem corporal em estudantes do curso de educação física. Revista Brasileira de Educação Física e Esporte. 2010;24(2):28592.

18. O'Brien KS, Hunter JA, Banks M. Implicit anti-fat bias in physical educators: physical attributes, ideology and socialization. International journal of obesity. 2007;31(2):308.

19. Bosi MLM, Luiz RR, Uchimura KY, Oliveira FPd. Comportamento alimentar e imagem corporal entre estudantes de educação física. J bras psiquiatr. 2008;57(1):28-33.

20. Coqueiro RdS, Petroski EL, Pelegrini A, Barbosa AR. Insatisfação com a imagem corporal: avaliação comparativa da associação com estado nutricional em universitários. Rev psiquiatr Rio Gd Sul. 2008;30(1):31-8.

21. Saikali CJ, Soubhia CS, Scalfaro BM, Cordás TA. Imagem corporal nos transtornos alimentares. Archives of Clinical Psychiatry. 2004;31(4):164-6.

22. Cash TF, Deagle III EA. The nature and extent of body-image disturbances in anorexia nervosa and bulimia nervosa: A meta-analysis. International Journal of Eating Disorders. 1997;22(2):107-26.

23. Silva DAS, Pereira IMM. Estágios de mudança de comportamento para atividade física e fatores associados em acadêmicos de educação física. Revista Brasileira de Atividade Física \& Saúde. 2010;15(1):15-20.

24. Romaro RA, Itokazu FM. Bulimia nervosa: revisão da literatura. Psicol Reflex Crit. 2002;15(2):407-12.

25. Moreira HdR, Nascimento JVd, Sonoo CN, Both J. Qualidade de vida no trabalho e perfil do estilo de vida individual de professores de Educação Física ao longo da carreira docente. Motriz Journal of Physical Education UNESP. 2010:900-

\section{OBSERVAÇÕES}

Os autores declaram não existir conflitos de interesse de qualquer natureza.

O autor Ricardo Pablo Passos é bolsista da CAPES. 